\title{
ASENTAMIENTOS ARQUEOLÓGICOS DEL MUNICIPIO DE LAS Choapas, Veracruz
}

\author{
María de Lourdes Hernández Jiménez
}

Resumen: Los recorridos extensivos que se derivan de la supervisión de obras sísmicas, paradójicamente suelen ser coyunturales para el conocimiento de una región, como es el caso de la obra de exploración de hidrocarburos, en la cuenca media del río Uxpanapa, cuya cobertura nos permitió registrar una serie de asentamientos prehispánicos que datan desde el preclásico olmeca hasta el Posclásico. Esta región, al estar ubicada en el centro del istmo y contener una red hidrológica amplia, permitió el desarrollo y los movimientos poblacionales entre la costa de Golfo y del Pacífico.

Palabras claves: patrón de asentamiento, áreas de cultivo, calzadas, terraplenes, juego de pelota, centros ceremoniales.

Enviado a dictamen: 03 de abril de 2012

Aprobación: 28 de mayo de 2012

Revisiones: 1

Mtra. María de Lourdes Hernández Jiménez, Arqueóloga y Maestra en Ciencias de la Educación y Restauración en arquitectura prehispánica. Profesora Investigadora del Centro INAH Veracruz. Temas de especialización: Estudios regionales de asentamientos arqueológicos en el sur de Veracruz. Correo electrónico: loheji@hotmail.com.
Abstract: The extensive routes derived from the monitoring of seismic works, paradoxically tend to be relevant to the knowledge of a region, as it is the case of the works of exploration of hydrocarbons, in the mid basin of the Uxpanapa River, whose coverage has allowed us to register a number of pre-Columbian settlements dating from the pre-classic Olmec through to the Postclassic; This region being located in the center of the isthmus and contain a comprehensive hydrological network, it allowed the development and population movements between the Gulf and Pacific coast.

Keywords: settlement patterns, farming areas, roads, embankments, ballcourt, ceremonial centers.

$\mathrm{E}$ n los últimos años, en la región sur de Veracruz, específicamente en las colindancias entre el municipio de las Choapas y los límites de los estados de Tabasco y Chiapas, se han realizado recorridos arqueológicos extensivos surgidos a raíz de la ejecución de algunas obras públicas. El interés de la extracción de hidrocarburos en esta zona ha dado lugar a que las compañías exploradoras de petróleo se coordinen con el INAH para la salvaguarda del patrimonio arqueológico que pudiera verse afectado. Dentro de ese marco se han recorrido varias extensiones de terreno con la finalidad de registrar y proteger los 
sitios arqueológicos y al mismo tiempo conocer la historia prehispánica de ésta región.

Es así como los trabajos actuales de recorrido de superficie llevados a cabo en la región parten del compromiso de supervisar y verificar los trabajos de exploración que realiza la compañía Mexicana de Exploraciones S.A de C.V. (COMESA). Para que no altere ni afecte las evidencias arqueológicas, hemos implementado una metodología apropiada a éste tipo de actividad arqueológica que, al estar inmersa en una dinámica constante de ejecución, difiere un poco de las metodologías de investigación específica. De ahí que la aplicación de software y de sistemas de información Geográfica han sido fundamentales para la recolección, concentración y análisis de datos.

Pues bien, la región que nos ocupa se localiza en la cuenca media del río Uxpanapa hasta pie de monte de la Sierra Atravesada. Es un área que ha tenido poca atención desde la perspectiva de investigación arqueológica. Las obras de esta magnitud pueden ser útiles y contribuir al conocimiento de la arqueología regional; por ello no nos hemos limitado solamente a la supervisión sino que hemos estado abiertos a diversas líneas de estudio, a raíz de varios aspectos culturales relevantes que hemos estado documentando. Sin embargo, al carecer de sondeos arqueológicos por la modalidad del proyecto como Supervisión, la tendencia superficial de este recorrido limita en demasía conocer la asociación tiempoespacio. Aunado a ello, a pesar de la escasez de material arqueológico en superficie, debido a la poca visibilidad que dejan los amplios terrenos de pastoreo de ganado mayor y los aislados manchones de vegetación, algunos restos cerámicos con huellas de policromía y hachas de cobre nos llevan a ubicarlos temporalmente en las primeras centurias del Posclásico. No obstante, esto puede ser confirmado o modificado cuando se realicen excavaciones que aporten datos más precisos.

\section{El área de estudio}

El área de estudio se localiza en la porción sur del municipio de Las Choapas, Veracruz (ver figura 1). Es un área donde predomina un sistema de lomeríos cuyos plegamientos descienden de la estribación norte de la Sierra Atravesada. La prominencia natural que resalta es el Cerro Nanchital, que es la antesala para llegar al pie de monte de dicha Sierra.

Éstos sistemas de elevaciones son surcados por escurrideros y pequeños arroyos cuya agua desciende a las zonas bajas, donde se acumulan formando áreas anegadas y pantanosas. Sobre la ribera este del río Uxpanapa y oeste del Tancochapa hay zonas inundables que, a veces, crean lagunas permanentes o temporales irrigadas por ambos ríos durante la temporada de lluvia.

La vegetación es escasa debido al impacto de la actividad ganadera que ha incidido fuertemente sobre el ecosistema. La reducción de selva conduce inevitablemente a la disminución de especies faunísticas.

Ocasionalmente sobre los lomeríos y elevaciones se observan manchones de selva secundaria (acahuales). En la cuenca alta de los ríos Uxpanapa y TonaláTancochapa, en la zona montañosa, todavía es posible notar reductos de selva alta perennifolia debido a su difícil acceso. Es aquí donde se pueden aún apreciar especies como tigrillos, mazates, jabalíes, coyotes, tejones, mapaches, monos aulladores, naoyacas, boas ratoneras, tucanes, loros, pericos, gavilanes, águilas, entre otras especies.

Hidrológicamente esta región está constituida básicamente por los ríos Uxpanapa y TancochapaTonalá, así como una serie de ríos y arroyos pequeños que les tributan. El río Uxpanapa es la corriente principal de esta región, tiene su origen en la Sierra Atravesada, en el municipio de San Miguel Chimalapa, Oaxaca, y lo alimentan varios arroyos antes de confluir con el Coatzacoalcos. El Tonalá nace en la Sierra Madre de Chiapas, a $1000 \mathrm{~m}$ de altitud, posee una longitud de $300 \mathrm{~km}$ y la mayor parte de su recorrido sirve como 
límite entre Veracruz y Tabasco. Su dirección transcurre generalmente hacia el noroeste y es navegable en la época de estiaje en gran parte de su extensión, al igual que sus afluentes. El río Tonalá, en su curso superior, es conocido como río Tancochapa. Sus principales afluentes en territorio Tabasqueño son los ríos Zanapa, Blasillo y Chicozapote.

\section{Investigaciones en el área}

Son escasos los trabajos realizados en el área de estudio. Resaltan apenas unos pocos realizados de forma sistemática. Sin embargo, Frans Blom estuvo interesado en la región durante su permanencia de 1919 a 1922. Como trabajador de la compañía petrolera El Águila tuvo la oportunidad de encontrarse con algunos sitios y vestigios arqueológicos, dejando noticia sobre ellos en unas cartas que enviaba a sus padres (Blom, 1990).

Más tarde en los años '50 del siglo pasado, Phillip Drucker y Eduardo Contreras (1953), realizaron reconocimientos en la zona con el objetivo de definir la frontera y los límites orientales del área nuclear olmeca. Estos autores recorrieron la parte este de San Lorenzo tomando como referencias los ríos Coatzacoalcos, Uxpanapa y el Blasillo. Allí detectaron varios asentamientos que jerarquizaron de acuerdo a la extensión, número de estructuras y monumentalidad.

En las postrimerías de los años ochenta, Manuel Torres, del Instituto de Antropología de la Universidad Veracruzana, realizó trabajos de rescate en el Arroyo Pesquero, lugar donde los pobladores descubrieron de manera fortuita una gran cantidad de máscaras estilo olmeca, olmecoides, teotihuacanas, hachas pulidas de piedra verde, de piedra burda, pequeñas esculturas, fragmentos de espejo de pirita y fragmentos de un vaso estilo teotihuacano (Medellín, 1971).

Mediante trabajos sistemáticos recientes realizados en la zona del recorrido, como el Salvamento de la autopista Las Choapas-Raudales-Ocozocuautla,se registraron pocos asentamientos arqueológicos con arquitectura en dos tramos del trazo carretero. Uno se localiza sobre la carretera federal 180 (CoatzacoalcosVillahermosa) y, el otro, más al sur, en los límites del municipio de Las Choapas con el estado de Chiapas (Cortés, 2001). El Proyecto Arroyo Pesquero dirigido por Carl Wendt contempla el conocimiento sobre la organización doméstica y la economía política. Durante el proceso lograron registrar varios sitios y material arqueológico. Su reconocimiento abarcó un área de $330 \mathrm{~km}^{2}$ (Wendt, 2005). Los datos que aportan fueron verificados por nuestro equipo, aunque sin la suerte de encontrarlos. Quizá sólo se trataba de sitios cerámicos sin estructura, aunque varios de esos puntos se encuentran cerca de otros sitios que nosotros registramos con arquitectura.

Por otrolado, los trabajos de salvamento derivados de obras de estudios sísmicos (exploración de yacimientos petrolíferos de compañías contratadas por Pemex) alrededor del área son los denominados Herradura y Herradura Norte, en la periferia sureste (Cortés, 2008), y por el extremo norte y este, las supervisiones de "Rodador 3D" y "Rosario-Cabrito 3D", éste último ya dentro del estado de Tabasco (Heredia, 2008). Todos reportan la presencia de sitios de arquitectura de tierra y, excepcionalmente, de piedra, de distintas extensiones y número. En el de Herradura Norte se registró el sitio San Miguel de Allende, de arquitectura de piedra, que cuenta con varias piedras labradas. En una de ellas puede verse el rostro descarnado de un mono con un numeral siete al estilo de punto y barra. En otras piedras se aprecian maquetas de canchas, juegos de pelota asociados a manantiales, cascadas y terrazas.

\section{Los trabajos arqueológicos}

En el año 2009 se iniciaron los trabajos de recorrido y supervisión arqueológica en el área de la poligonal de la obra de exploración petrolera de COMESA. Los objetivos, además de registrar los sitios prehispánicos, consistían en conocer e identificar el patrón de asentamiento, la 
cronología, así como las relaciones socioeconómicas con otras regiones de áreas aledañas de Tabasco, Chiapas y Oaxaca, considerando esta parte como una región habitada por la familia lingüística zoque-mixe.

El área total del polígono abarca una extensión de $2069 \mathrm{~km}^{2}$ pero la primera parte del recorrido se concentró en un área de 372.47 km, al interior del mismo, al este del río Uxpanapa. La topografía del lugar está conformada mayoritariamente por lomeríos que van de los 5 hasta los $60 \mathrm{msnm}$, donde se concentra una densidad de localidades arqueológicas.

\section{Los sitios arqueológicos en el contexto del río Uxpanapa}

Los vestigios más tempranos que se han identificado datan del Preclásico temprano. Las evidencias nos remiten a la fase San Lorenzo (1200-900 a. c.), identificadas con los tipos cerámicos de Las Calzadas y El Limón inciso (Coe y Diehl, 1980). Otras muestras indican tiempos contemporáneos a La Venta. Este tipo de sitios son bastante frecuentes, sobre todo en las márgenes del rio Uxpanapa y en lomas de altura baja cercanas a arroyos, lagunas y áreas inundables. Indiscutiblemente ésta fue la ruta natural por excelencia debido a la cantidad de ríos que la atraviesan, que usaron los olmecas para llegar de las regiones de la Costa del Golfo al Pacífico y viceversa, con fines de intercambio comercial, político y religioso. La ubicación de los sitios fue estratégica para el enlace y el control de rutas, las mismas que fueron reutilizadas en tiempos posteriores. De ahí que para algunos restos arquitectónicos asociados a los materiales olmecas no se haya podido determinar su pertenencia a ese tiempo y grupo.

En general los restos arquitectónicos presentan un patrón que los ubica cronológicamente en periodos del Clásico Tardío y Posclásico en la región del Istmo. Sin embargo, no se descarta la posibilidad de que algunos lugares hayan sido repoblados y sus edificios tempranos reutilizados con ciertas modificaciones.
Los componentes arquitectónicos de los sitios registrados presentan características similares en materiales y sistemas constructivos a base de tierra. La mayoría de los conjuntos arquitectónicos de periodos tardíos se construyeron sobre la cima de lomas de alturas variables. Es en esos espacios donde se distribuyeron una serie de edificios posicionados estratégicamente para ocupar el terreno disponible. En los terrenos estrechos de las elevaciones, cuando hubo la necesidad de mayor cobertura, tuvieron que condicionar las laderas para construir otras estructuras.

El resultado fue un interesante patrón de asentamiento que incluye plazas localizadas sobre las partes más amplias y de menor altura. Con el fin de establecer las dimensiones espaciales requeridas para ceremonias o rituales, flanqueadas por los edificios principales, los conjuntos secundarios, como áreas residenciales u habitacionales, aparecen asociados a ellas. O bien, al carecer de espacios, se construyeron en lomas adyacentes que fueron enlazadas a través de terraplenes. En las lomas de mayor altura, generalmente asociadas y en estrecha relación con la plaza y las áreas residenciales, se colocaron uno o dos edificios a modo de vigía. Desde ahí se domina todo el paisaje que alcanza hasta las elevaciones de las sierras, al sur, y las llanuras del Golfo, al norte. Es posible que este punto en la loma funcionara como estación de comunicación a distancia y quizá también para resguardar y controlar el acceso al área. Al estar la plaza colocada en una loma de altura media (irebajada?), espaciosa, da la impresión de hallarse protegida por las demás que la rodean, sobre todo por la loma de gran altura que le sirve de guardián.

El sistema de lomeríos donde se recreaban los espacios urbanos fueron conectados por medio de terraplenes, lo cual les permitió crear una red de caminos sinuosos que facilitaron un tránsito ágil entre grupos arquitectónicos localizados sobre las lomas.

La distribución de las plataformas ante la escasez de terrenos, en la superficie de la loma, dan la impresión de que los espacios disponibles fueron utilizados al 
máximo, edificando en las orillas cerca de las pendientes, unas veces suaves, otras veces abruptas, con el fin de ganar espacio para el interior de los patios. Al parecer no procuraron construir plataformas para su ampliación. De esa forma se configuró el patrón de distribución de los edificios, los cuales se fueron construyendo según dictara la forma natural de las elevaciones.

La forma de las plazas era por lo general de planta trapezoidal. Se colocaban, alineados en ambos extremos, varios edificios de planta ligeramente alargada, uno junto al otro, quedando muy cerca de la orilla de la colina. En cada extremo, un montículo mayor de planta circular o rectangular y en el lugar opuesto otro más, generalmente rectangular, que a veces quedaba abierto. El inseparable juego de pelota en forma de "T" se adosaba en uno de sus lados. La orientación de las plazas la hemos observado en dos variantes, E-W y N-S, con ligeras desviaciones al este.

Las áreas administrativas, residenciales o habitacionales en su mayoría, se arreglaron en patios cerrados o abiertos y otros edificios adjuntos asociados según permitiera el terreno. Las áreas abiertas se redujeron en pos de las construcciones. Esto se hace evidente en el momento de transitar entre edificios, ya que hay que hacerlo entre ellos o muy cerca de la orilla de la loma. El estilo arquitectónico predominante en los edificios es de planta alargada o rectangular, contando con algunas de planta circular.

En el área de lomas extendidas, donde se alcanzan las llanuras, hay excepciones en cuanto a la distribución de los edificios. Es ahí donde se contaba con espacios suficientes. Los constructores prehispánicos aprovecharon el terreno solventando todo tipo de construcciones en esos espacios, pero sin perder de vista el esquema y patrón urbano de plazas plenamente identificadas al interior de los lomeríos.

Este patrón de asentamiento caracteriza y unifica al área este de la cuenca media del Uxpanapa. Resalta fuertemente la forma en que se adaptaron a los espacios reducidos, colocando terraplenes ente loma y loma para caminar ligero y conducirse cómodamente a sus centros principales, a sus viviendas o campos de cultivo.

Esta conjugación entre lo artificial y natural, modificando unas veces o adaptándose otras, recreó paisajes armoniosos, interesantes, que dieron como resultado la característica particular del grupo que se desarrolló en la región este del río Uxpanapa, cuya cobertura parece abarcar un territorio que va del pie de monte hasta la llanura de la costa del Golfo.

\section{Los principales sitios arqueológicos}

Nos enfocaremos a describir tres sitios que representan, según nuestro punto de vista, los centros rectores y de mayor alcance por su extensión y numero de estructuras. Dos de ellos se encuentran localizados en la ribera del los ríos Uxpanapa y Tancochapa lo que les da un carácter estratégico, a manera de punto de control. El otro se encuentra al interior, quizá con la misma función que para los de tierra adentro. Estos tres se ubican en un punto donde los ríos mencionados alcanzan una relativa cercanía.

\section{Sitio El Diamante}

Este sitio arqueológico, denominado así por encontrarse en el Rancho El Diamante, se localiza al 2 km aproximadamente al sur de la ciudad de Las Choapas y a pocos metros de la comunidad de San José del Carmen. Fue una ciudad rivereña sobre el lado oeste del río Tancochapa, asentada sobre un terreno de lomas medias suaves y ondulantes que discurren en un eje este-oeste hacia la margen rivereña, rodeadas por bajos inundables. En algunas secciones se ve interrumpido, de forma más o menos gradual, por un cinturón de acahual denso que precede a una ligera barranca, la cual se enfila hacia el área de inundación del río Tancochapa.

El asentamiento se extiende sobre 58 ha, donde se han conservado 126 edificios distribuidos en toda el área: la Plaza principal, patios, zonas residenciales 
y varias terrazas habitacionales. En la zona de mayor concentración, con 87 estructuras, se encuentra la Plaza principal que se localiza un kilómetro más o menos al noroeste del río Tancochapa y ubicada en lo más elevado del área. En este conjunto se registraron además de la Plaza, siete patios y una cancha de juego de pelota (ver figura 2).

La Plaza principal mide 73 metros de largo por 18 metros de ancho. Orientada norte-sur con desviación al este y flanqueada por dos largueros asimétricos, el del extremo oeste es más largo que el del este. Su longitud queda compensada por dos estructuras pequeñas en cada extremo, una de las cuales es el cabezal del juego de pelota (ver imagen 1). La estructura que funciona como cabezal principal se ubica en el extremo norte, de planta circular, aunque actualmente se encuentra un poco deteriorada hacia el extremo sur. Lo cierra un larguero que excede del espacio de la Plaza, aunque este excedente pareciera como un adosamiento posterior. Paralelo a esta plaza, al este, se construyó la cancha del juego de pelota en forma de " $\mathrm{T}$ ".

La plaza se encuentra rodeada por áreas administrativas y residenciales a juzgar por la distribución y dimensiones de los edificios formando patios y agrupaciones. Más allá de esta concentración, hacia los ramales de este sistema de lomeríos, se encuentran diseminados algunos otros montículos quizá de orden habitacional.

\section{Sitio Rivera del Carmen}

Este sitio localizado en la margen izquierda del rio Uxpanapa se ubica al suroeste de la ciudad de las Choapas, Veracruz, a poco menos de 20 kilómetros, en la comunidad de Rivera del Carmen.

El asentamiento arqueológico está emplazado cuesta arriba de la pequeña sierra de colinas medias que bordean el recodo del río Uxpanapa y en las faldas de ella, en una extensión de 25 ha. Ésta pequeña cordillera se eleva en la margen izquierda del río
Uxpanapa creando un meandro notable en su cauce, al tiempo que se generan grandes espacios de pantanos y zonas inundables alrededor del macizo colinar. Las estructuras se construyeron sobre algunas aristas medianas y altas (más o menos de 70 metros). Estas colinas están cortadas por grandes y profundas barrancas de donde nacen corrientes de agua dulce y arroyos perennes que desembocan a la laguna Rivera del Carmen y al rio Uxpanapa.

Este sitio cuenta con 25 estructuras de tierra. Dispuestas a lo largo de las prominencias de las colinas que descienden de la cordillera, se modifican a través de nivelaciones o terrazas, sobre las cuales construyeron edificios formando plazas abiertas y patios. Lo intrincado de las lomas y la manera natural en que éstas se conectan forzaron a los antiguos pobladores a orientar sus edificios como permitió el reducido espacio natural, lo que le dio una característica especial al asentamiento, con corredores y pasos estrechos, así como espacios abiertos con barrancas profundas a sus costados. La dispersión de los edificios puede definirse en 3 conjuntos, cada uno correspondiente a cierta zona de la cordillera sobre la que están emplazados y separados por barranquillas y arroyos.

El conjunto principal está constituido por 13 montículos, una terraza, y unido por un terraplén. La Plaza Principal, orientada en sentido este a oeste, de planta casi trapezoidal, es lo más significativo del sitio. Está conformada por siete montículos, dos de ellos alineados en su costado norte, fungiendo como largueros delimitantes. En sus extremos este y oeste lo cierran montículos de planta rectangular, uno de ellos, el del lado oeste, es el cabezal mayor. Este montículo principal de la plaza destaca por el hecho de encontrarse en el desnivel natural de la ladera sobre una terraza acondicionada, lo que le confiere una altura considerable con respecto al resto de los edificios de la plaza (6 metros, aproximadamente), por lo que consideramos fungió como cabezal principal (ver imagen 2). Los demás montículos se despliegan 
sobre los ramales que descienden de la cordillera, ya sea formando conjuntos de patios, juegos de pelota o terrazas (ver figura 3).

La ubicación de la plaza, construida en un espacio elevado y relativamente amplio, en un corredor natural con ligeros desniveles topográficos delimitado por dos profundas barrancas, le otorga una vista incomparable y domina un espectacular paisaje, por un lado la laguna y el río (al norte) y, por el otro, las zonas inundables del sur.

\section{Sitio El Chichón-Acapulco}

El sitio arqueológico se localiza a $16.5 \mathrm{~km}$ al suroeste de Las Choapas, cerca de la población del mismo nombre, dentro de los terrenos del Rancho Acapulco. Por su extensión, dimensiones y número de edificios, ha sido considerado como el centro primario que cohesionó el área al interior este del Uxpanapa. Se halla emplazado sobre una zona de lomeríos bajos con superficies suficientemente amplias y llanas, rodeado por arroyos intermitentes y escurrideros. Ocupa una extensión de 288.73 ha, con un total de 319 estructuras seccionadas en 11 conjuntos que conforman 5 plazas, 13 patios y 4 Juegos de pelota. Las alturas de las estructuras oscilan entre los 2 y 15 metros (ver figura 4).

La mayor concentración se encuentra en el conjunto principal. Cuenta con una Plaza de planta trapezoidal, orientada este-oeste, limitada en sus cuatro lados por estructuras largas. Se trata de un área cerrada que mide $96 \mathrm{~m}$ de largo por $64 \mathrm{~m}$ de ancho (ver imagen 3). Existen solo dos accesos, uno en la esquina noroeste y otro en la esquina noreste. En esta esquina y al frente del montículo mayor, a unos $10 \mathrm{~m}$, hay un pequeño altar de unos $50 \mathrm{~cm}$ de altura y de planta circular. En el costado norte se adosó el juego de pelota, cuyo edificio largo cierra el extremo lateral norte de la plaza y funciona como paramento de la cancha del juego de pelota del tipo doble "T".
Hacia el noreste y noroeste se encuentran otros edificios de dimensiones considerables que posiblemente funcionaron como centros administrativos y de gestión. En una loma contigua, al oeste, se concentra otra serie de estructuras. Quizá se trate del área residencial, conformada por patios.

Al norte se observa otra plaza pero con características diferentes. Sus edificios largos están alejados unos de otros haciendo el espacio muy abierto. También cuenta con un juego de pelota: la prominencia que resalta ahí es el montículo principal, de planta circular, con una altura de 13 metros.

Hacia el este de la plaza Principal, se construyeron otras plazas de dimensiones menores emulando la principal. Alrededor de ellas se construyeron otros conjuntos de edificios habitacionales y patios.

La monumentalidad y la extensión le dan una categoría de ciudad de primer orden. Desde allí se gobernaba el destino de la población y se controlaban los productos locales y foráneos. Asentado sobre unas lomas bajas extendidas constituye la excepción en el entorno de los demás asentamientos.

Los sitios descritos no son los únicos, ya que existen un buen número de sitios menores que los van enlazando con terraplenes, interlomas o puentes de madera si los interrumpen los bajos inundables. En el caso de los sitios El Diamante y Rivera del Carmen, ubicados en las márgenes de los ríos Tancochapa y Uxpanapa respectivamente, existen otros asentamientos que los conectan y van delineando una probable ruta terrestre que unía ambos ríos.

A partir del sitio El Chichón-Acapulco, hacia el sur, se despliegan un sinnúmero de sitios que coronan las lomas de la zona, entre los que se encuentran otros sitios secundarios que cuentan con plazas de dimensiones menores, patios residenciales y áreas habitación. Uno de los que vale la pena resaltar es el sitio Los Basamentos por la forma en que construyeron sus áreas de residencia y su centro ceremonial. Trabajaron las lomas que lo sustentan para darle la apariencia de unos grandes 
basamentos, estilo Acrópolis y sobre ella construyeron los edificios que forman patios residenciales. El pie de estos dos basamentos, se nota que estuvo rodeado de agua, pues recuerda las áreas inundadas alrededor de los castillos medievales que servían para protegerlos y controlar los accesos. Pudiera darse el caso de que fueran también defensivos o al mismo tiempo para contener y almacenar agua para uso diario, o quizá para la cría de peces u otras especies acuáticas.

Hacia el sur existen otros sitios compuestos por estructuras menores, como por ejemplo Palma sola, La Comarca, El Cementerio, La Canchas, La Cantera y La Cordobesa, que forman un núcleo compacto donde se congregaron un numero cuantioso de edificios, ya sea formado plazas, patios abiertos, juegos de pelota, u otra serie de edificios en conjunto, solos sobre el tope de la loma, o en sus laderas. Lo relevante de esta nucleación es la facilidad para transitar de una loma a otra a través de terraplenes artificiales o pasos acondicionados. En este conglomerado no es posible distinguir en qué momento estamos pasando de un territorio a otro. No es visible una línea divisoria, sino que, de pronto, aparece frente a nosotros una plaza menor, custodiada por una loma de mayor altura que sirve de vigía y protección: es cuando nos damos cuenta de que estamos en otra unidad urbana. Al costado oeste de estos sitios hay otros asentamientos distribuidos en una serie de lomas ocupadas por edificios pequeños en altura y volumen, interrumpidas por arroyos intermitentes, bajos inundables y escurrideros. Al final, cesadas por zonas bajas de la ribera del río Uxpanapa, se llega al pie de monte del cerro Nanchital, alfil natural que, por el momento, es nuestro límite sur y enmarca el área de recorrido.

La concentración masiva de los sitios donde se observan centros cívicos ceremoniales, áreas residenciales, conjuntos de patios en lomas conectadas por terraplenes, ya sea con pocas estructuras o sólo la nivelación de la cúspide, nosimposibilita por el momento determinar con precisión su jerarquía. Arbitrariamente los denominamos con cierta categorización basada en datos de campo que podemos inferir por su tamaño y extensión.

Por esas rutas se ven los vestigios de los asentamientos arqueológicos interesantes no sólo por ser desconocidos en la literatura arqueológica, sino por las características del medio donde se localizan. Las lomas se encuentran bordeadas por áreas de escurridero y bajos inundables. Este escenario le confiere relevancia desde el punto de vista de la importancia que tuvieron estos humedales en la vida de los antiguos pobladores.

La utilidad de esos terrenos bajos ha sido planteada tanto para las zonas mayas (Covarrubias, 2001; Culbert, 2005) como para el área de la selva El Ocote, en Chiapas (Domenici, 2009). Se cree que, para asegurar la subsistencia de una población elevada, los bajos inundables fueron la alternativa para incrementar la producción agrícola.

En nuestra área de recorridos hemos registrado camellones que nos sugieren su uso para la agricultura, tal como se ve hoy día, y están en zonas bajas aledañas a los conjuntos arquitectónicos. Tomando en cuenta que la topografía del la zona es muy accidentada y que tenían pocas alternativas de tierras llanas para cultivo, aprovecharon bastante bien los terrenos de las laderas y los bajos relativamente amplios acondicionándolos con el fin de emplearlos para el cultivo de forma intensiva.

En las áreas interlomas, donde había la posibilidad de apoyarse en terrenos relativamente amplios y con muy ligeras pendientes, se levantaban pequeños espacios de terreno a modo de camellones para aprovechar los escurrimientos provenientes de las lomas. Eran canalizados hacia un arroyo en la vertiente, lo que permitía humedad suficiente para irrigar el cultivo. La mayoría de este tipo de espacios los hemos observado asociados a centros ceremoniales.

Otra modalidad observamos en las áreas extensas inundables, donde de igual modo levantaban una porción de terreno como camellones y los separaban por canales. Presumimos que eran terrenos que no llegaban a 
inundarse totalmente, cuyos canales funcionaban como drenes en épocas de mucha agua, evitando dañar las siembras. Este tipo de terreno se observa más alejado de la gran urbe donde existen grandes separaciones entre lomas.

\section{Materiales arqueológicos}

Los materiales arqueológicos son uno de los elementos que nos acercan temporalmente a los restos arquitectónicos antiguos y por eso son indispensables para plantear fechas relativas, pero surgen dificultades cuando están muy deteriorados y reducidos como es el caso que nos ocupa. A diferencia de la alta densidad de sitios que hemos registrado, los artefactos arqueológicos son escasos, debido principalmente a alta acidez del suelo y las actividades ganaderas de la región, cuyas grandes extensiones de pasto cubren el suelo impidiendo verlos. También por los reductos de selva es difícil hacer la recolección que hacemos en los puntos de erosión, caminos, cortes de terrenos, y donde hay áreas de cultivo que, por cierto, son pocas.

En varios de los sitios que se encuentran sobre la rivera del Uxpanapa se recolectó cerámica diagnóstica de dos temporalidades básicamente, siendo la del periodo Clásico la de mayor densidad. Posiblemente la ocupación en la zona se haya prolongado hasta los primeros tiempos del Posclásico. Las muestras del Preclásico se encontraron en zonas bajas, cerca del río y lagunas, o en las faldas de los lomeríos, contextos muy característicos de esos asentamientos tempranos. Los pobladores del Clásico-Posclásico, por el contrario optaron por vivir en las cimas de las lomas donde se reconocieron grandes y pequeños conjuntos arquitectónicos.

Los tiempos que podemos manejar de acuerdo al tipo cerámico Naranja fino de núcleo negro y compacto son los que están estrechamente relacionados con sitios del Clásico Tardío documentados para la cuenca del Coatzacoalcos. Abundan también tipos burdos utilitarios, mínimamente se observan del Tipo Naranja Fino Deleznable característico de los lomeríos internos del Istmo, asociados a estructuras monumentales. No obstante, habría que hacer más minucioso nuestro análisis debido a la presencia de otro tipo que nos lleva hasta el Posclásico como el Crema Fino con núcleo oscuro, cuyo acabado y decoraciones polícromas nos inducen a pensar que tiene una liga con la tradición mixteca.

Algo que nos llama la atención es la presencia de hachas de cobre, a veces fragmentadas, otras completas. Las formas y tamaños difieren, así como su grosor, pero predominan las de cuerpo muy delgado. Desafortunadamente, sus muestras se encuentran en posesión de los habitantes.

La presencia de éstos objetos en la región nos situaría en una ruta de comercio con gente proveniente del occidente desde el Clásico Tardío que tuvo como centros importantes expulsores el sur de Michoacán y algunas zonas de Guerrero, así como de grupos del Posclásico de la Depresión Central y de Los Altos de Chiapas. Esto supondría relaciones más allá del hinterland istmeño Veracruzano y Oaxaqueño.

Si enlazamos la información de las muestras cerámicas con las hachas de cobre, tendríamos que replantearnos la ubicación temporal de los asentamientos en la cuenca media del río Uxpanapa. Por ahora no podemos determinar si la arquitectura manifiesta en la superficie actual sea de la ocupación de finales del periodo Clásico o de alguna fase del Posclásico debido a la limitada información que contamos con referencia a otros indicadores temporales y a estudios de campo más profundos.

\section{Comentarios Finales}

La región del Uxpanapa es un área con una amplia red hidrológica que fue fundamental y determinante para la conexión de regiones de la costa del Golfo y del Pacífico, así como para el interior del istmo, desde tiempos 
mesoamericanos. La riqueza en recursos naturales contribuyó también al desarrollo de asentamientos humanos y el tránsito de personas o bienes a través de los ríos principales y los ramales fluviales. Entre ambas regiones hubo múltiples y distintas interrelaciones que fueron muy variadas y extensas.

Fue el escenario de muchas oleadas y tránsitos de diversos grupos en diferentes tiempos. Constituyendo el corazón de la familia lingüística zoque-mixe, fue una de las rutas principales que utilizaron desde época temprana; del Soconusco vinieron grupos Mocayas y, un poco después, habitantes de San Lorenzo de la costa del Golfo, seguidos por expediciones de teotihuacanos y otros grupos nahuas en el Clásico. Grupos de la parte occidental de Chiapas, de zapotecos y mixtecos, también realizaron travesías por las rutas de esta región para alcanzar la costa del Golfo durante el Posclásico.

Ruta eminentemente olmeca en sus tiempos temprano y medio, figuran a la cabeza San Lorenzo y después La venta como orígenes y destinos de esas circulaciones constantes. La presencia de sitios de este periodo da cuenta de ello, la mayoría asentados en las márgenes del río Uxpanapa o en áreas próximas a las lagunas y áreas bajas inundables.

La densidad de sitios arqueológicos registrados tanto en las riveras como en los lomeríos al interior del área de estudio, que datan de tiempos posteriores a lo olmeca, señala el auge que retomó la región como ruta ístmica ya que, al parecer, se trata de un área conformada por varios centros secundarios y cohesionados por algunos centros rectores fuertemente vinculados con dos de los cuerpos de agua más importantes de la región, los ríos Uxpanapa y Tancochapa-Tonalá.

Siendo ambos de mayor cauce, se entiende que la posición en ambas márgenes de los sitos El Diamante y Rivera del Carmen, fue de relevancia para el control y vigilancia. Considerando además que en esta parte los ríos se ubican relativamente cerca, delimitan una franja terrestre estrecha, lo que permitió y facilitó el tránsito de personas y mercancías. Ambas ciudades mantuvieron el papel de centros de control para la llegada y salida de productos a las regiones del Golfo y el Pacífico puesto que los mencionados ríos conectan a las regiones de los Chimalapas-Uxpanapa con los de los Tuxtla-Coatzacoalcos y a los de la región occidental de Chiapas con la región de la Chontalpa y la península de Yucatán. Por lo que la posición de los sitios El Diamante y Rivera del Carmen, en ambos extremos de esa tierra firme estrecha, sirvió para transitar de un lado a otro, siendo muy probable que esta parte haya funcionado como bifurcación de la ruta según el destino que se llevara.

$\mathrm{Al}$ parecer este conjunto de ciudades centrales y dependientes, pudieron haber estado insertas dentro de un sistema regional de relaciones socio-políticas y económicas, lo que permitió cierto dominio de los canales de comunicación terrestre y acuático y, consecuentemente, del intercambio de 1 productos foráneos y locales.

En este sentido, suponemos que El Diamante y Rivera del Carmen fueron los centros principales con posiciones estratégicas y posiciones de poder religioso, administrativo y político que dominaron la zona ribereña, mientras que El Chichón-Acapulco dominó hacia la parte sur de tierra adentro.

\section{Bibliografía}

Coe, Michael y Richard Diehl (1980), In the land of the olmec. The Archaeology of San Lorenzo Tenochtitlan, Austin, Texas, USA: University of Texas Press.

Cortés Hernández, Jaime (2001), Salvamento Arqueológico Las Choapas-Raudales-Ocozocoautla. Informe Técnico Final. México: Archivo Técnico INAH Veracruz.

Cortés Hernández, Jaime y Raúl Jiménez (2008), Programa de Supervisión Arqueológica del proyecto de Exploración Sísmica Herradura 3D, así como del resultado de su ampliación, Informe Técnico Final, México: Archivo Técnico, Instituto Nacional de Antropología e Historia. 
Covarrubias Reyna, Miguel (2001), "Reconocimiento arqueológico en la región oriental del estado de Campeche", en Temas Antropológicos, Revista científica de investigaciones regionales, vol. 23, núm. 2, México: Universidad Autónoma de Yucatán. Facultad de Ciencias Antropológicas, pp. 181-200.

Culbert T., Patrick (1995), Antigua agricultura Maya en tierras pantanosas. www.famsi.org/spanish/reports/ author.php?author=c $-d$. [consultado: el 20 de marzo de 2011].

Daneels, Annick (2004), "Máscaras de Piedra de estilo teotihuacanas en la costa del Golfo", en Ma. Elena Ruíz G y Arturo Pascual S. (Editores), Memoria de la segunda mesa redonda de Teotihuacán, La costa del Golfo en tiempos teotihuacanos: propuestas y perspectivas, México: INAH, pp. 393-426.

Domenici, Davide (2009), "Continuidades, discontinuidades e interacciones culturales en el desarrollo cultural prehispánico de la selva El Ocote, Chiapas", en Thomas A. Lee, Davide Domenici, Víctor M. Esponda J. y Carlos U. Del Carpio P. (Coords.), Medio ambiente, antropología, historia y poder regional en el occidente de Chiapas y el Istmo de Tehuantepec, Colección Selva Negra, México: UNICACH. Chiapas, México, pp. 137-154.
Drucker Philip y Eduardo Contreras (1953), "Site Patterns in the Eastern part of olmec territory", en Journal of the Washington Academy of Science, XLII, Baltimore.

Frans, Blom(1990), Enellugardelosgrandesbosques, Epistolario de 1919-1922 y diario de dos expediciones), Traducción de Víctor M. Esponda, Charlie Cray y Eva Yul, México: Instituto Chiapaneco de la Cultura-Instituto Nacional de Antropología e Historia- Programa cultural de las fronteras, Tuxtla Gutiérrez Chiapas.

Heredia B, Luis (2007), Patrón de asentamiento en el sur de Veracruz, Tesis de Maestría. México: ENAH-INAH.

Hernández Jiménez, Lourdes, et.al (2007), Supervisión arqueológica Almagres 2D. Bloque I y II. Informe Técnico Final, Archivo Técnico INAH, México.

Hernández Jiménez, Lourdes, et.al (2008), Supervisión arqueológica Almagres 2D. Ampliación Chalca. Informe Técnico Final. Archivo Técnico INAH, México.

Hernández Jiménez, Lourdes, et.al (2010), Inspección Emergente en las obras de Estudio de Reflexión Sísmica Tepetate NW-El Plan-Los Soldados. Almagres 2D. Informe Técnico. Archivo Técnico INAH, México.

Wendt, Carl (2007), Reconocimiento Arqueológico en la Región del Río Pesquero, Veracruz México, Temporada 2005, Informe Técnico presentado al Consejo de Arqueología-INAH, México. 
Figura 1. Ubicación del área de estudio dentro de la cuenca media del río Uxpanapa

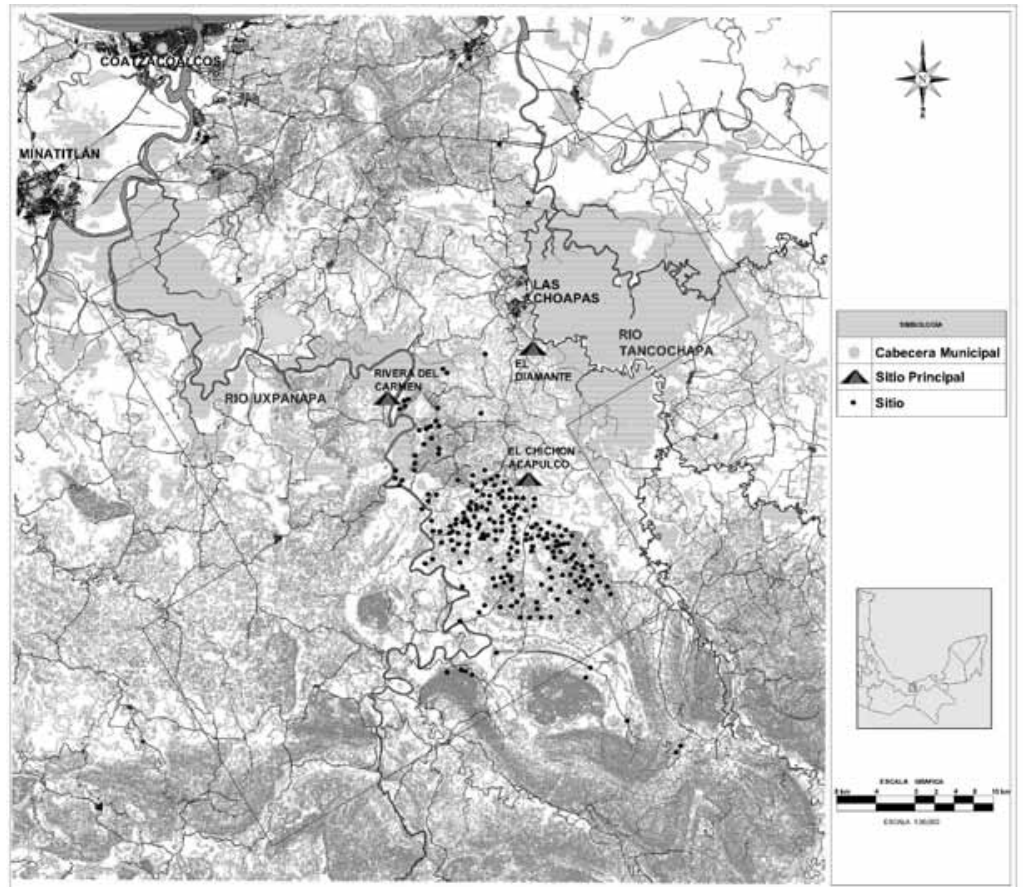

Figura 2. Plano del área principal del sitio El Diamante

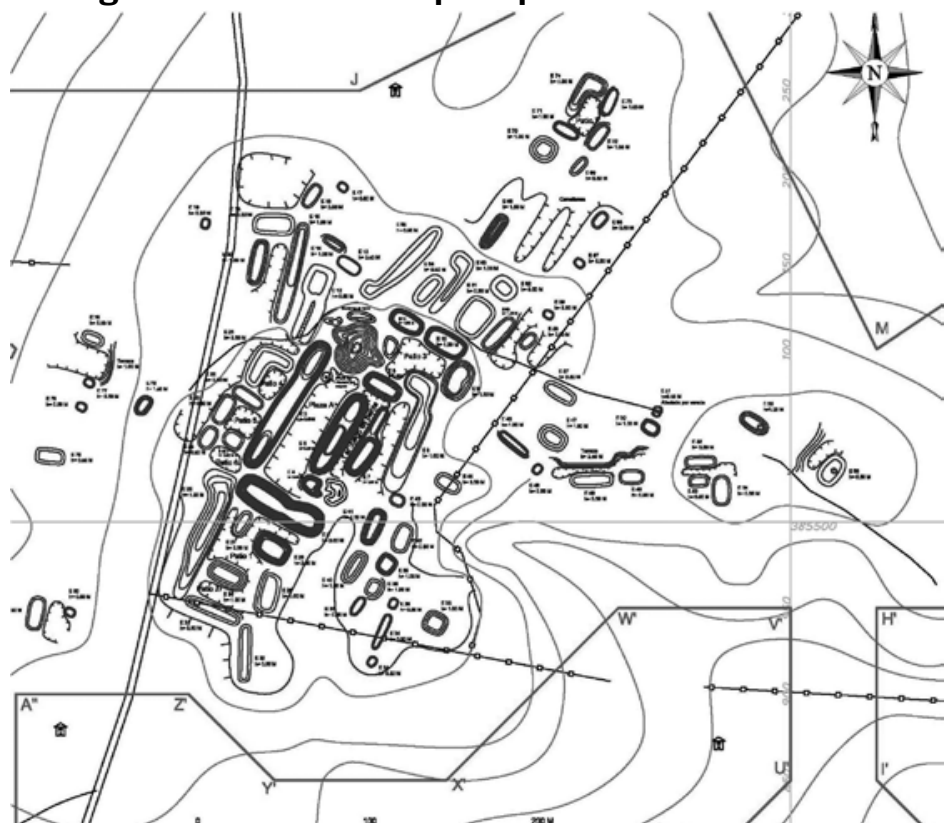


Figura 3. Ceremonial y conjuntos principales del sitio arqueológico de Rivera del Carmen

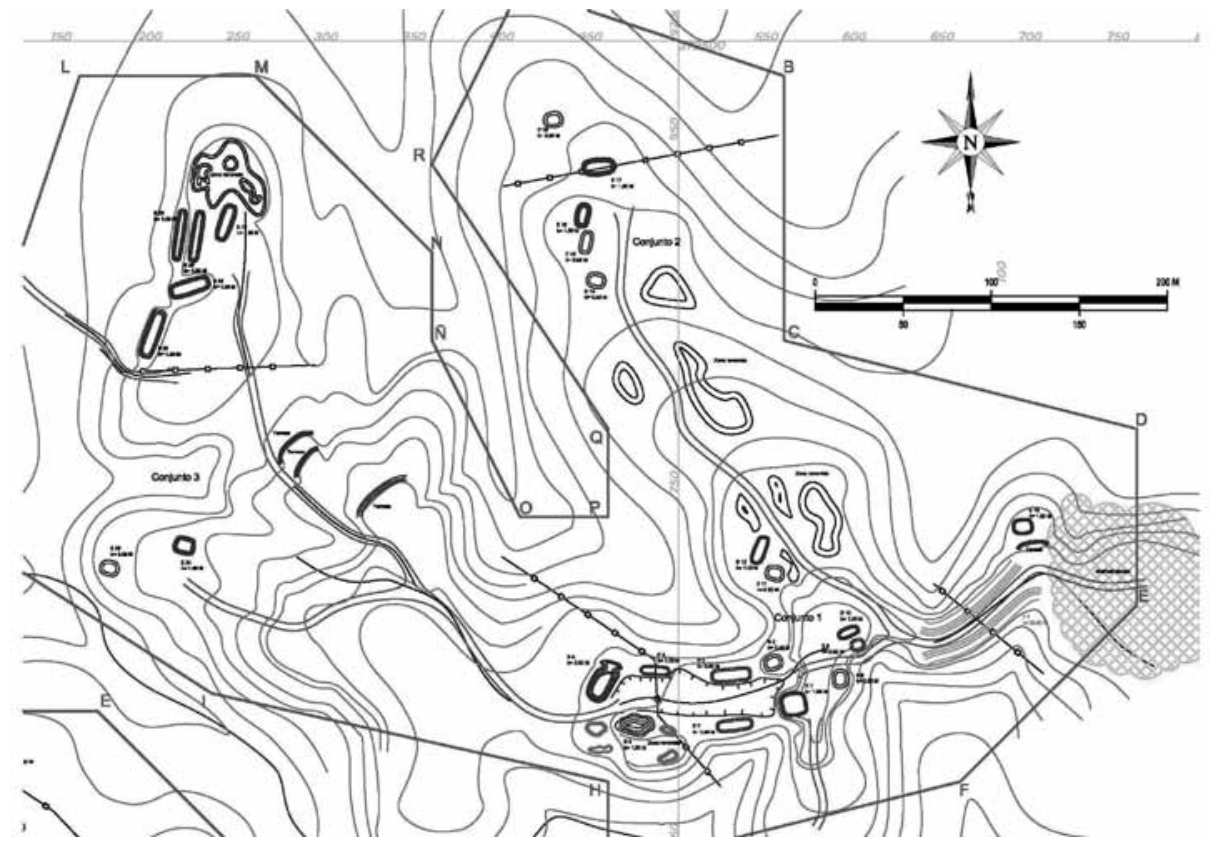

Figura 4. Plaza y Conjuntos principales del Sitio arqueológico El Chichón-Acapulco

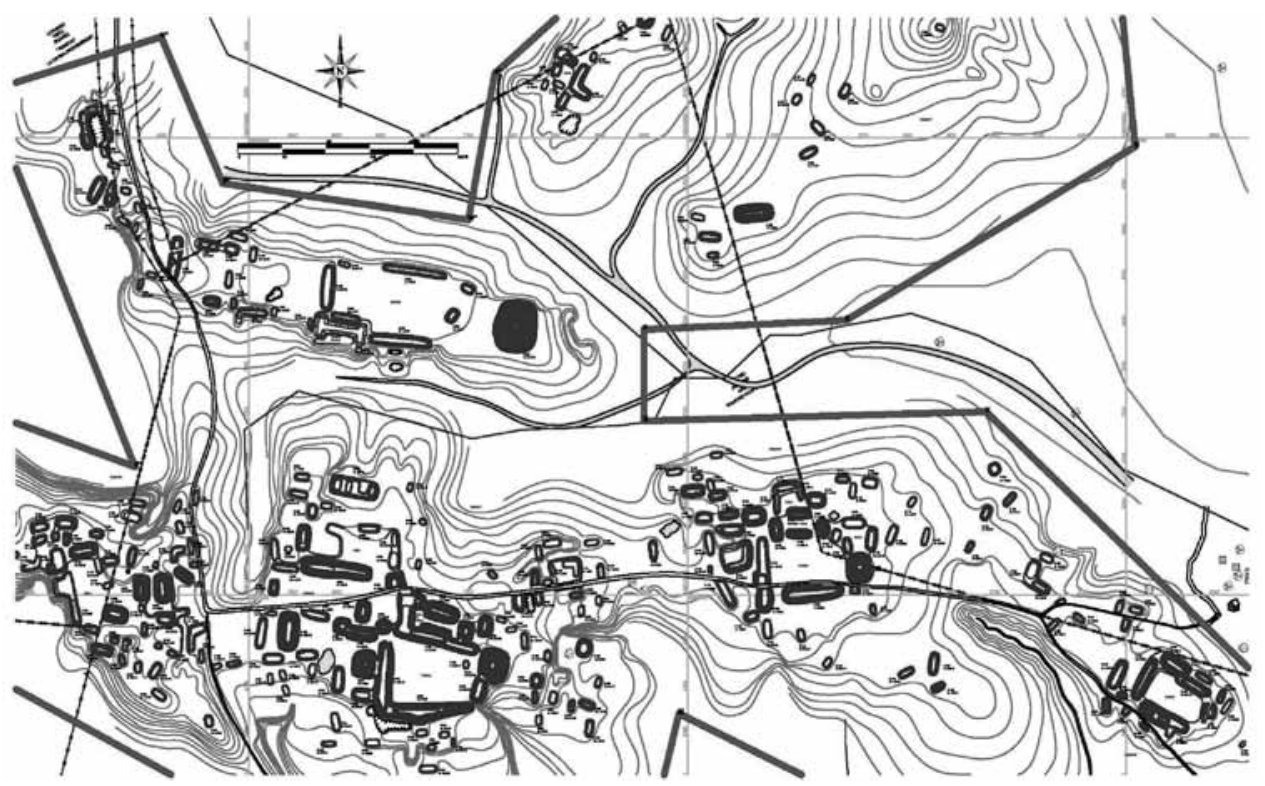


Figura 5. Ubicación de los sitios El Diamante, Rivera del Carmen y El Chichón-Acapulco y otros sitios al sur, entre los ríos Uxpanapa y Tancochapa-Tonalá

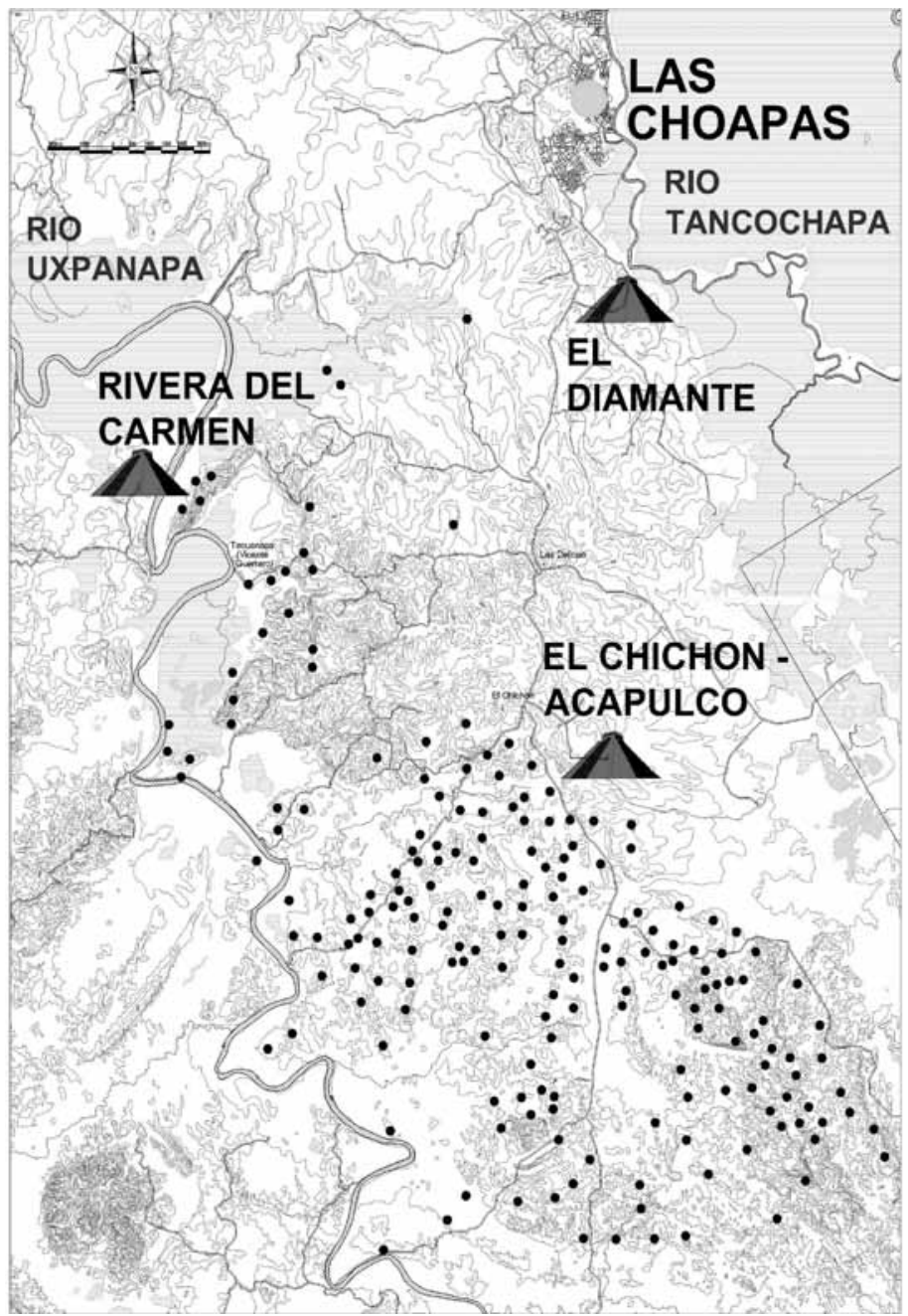


Foto 1. Plaza principal del sitio El Diamante; vista de suroeste a noreste. Al fondo se observa la Estructura que la delimita cubierta por un árbol

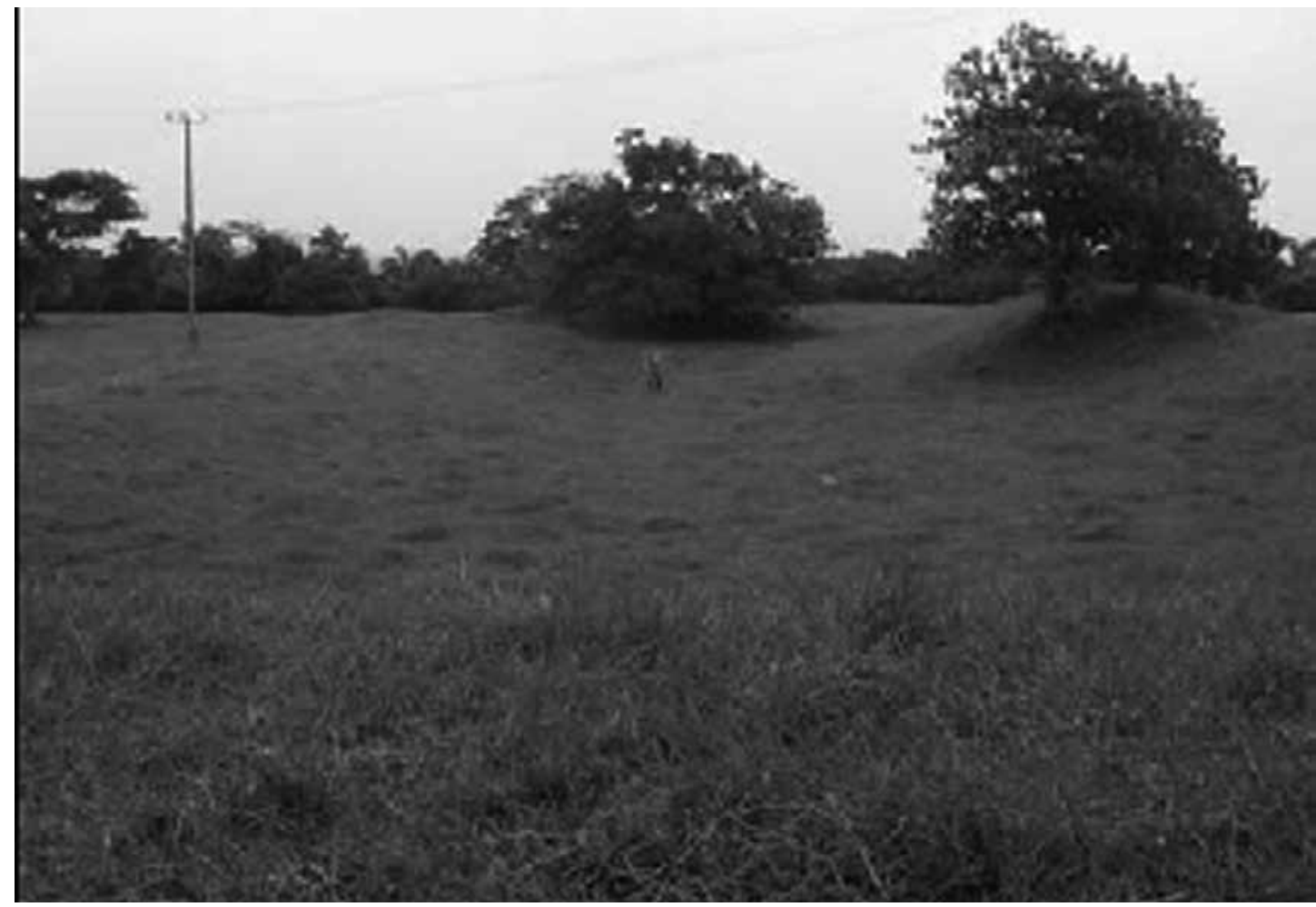

Fioto 2. Plaza principal del sitio Rivera del Carmen

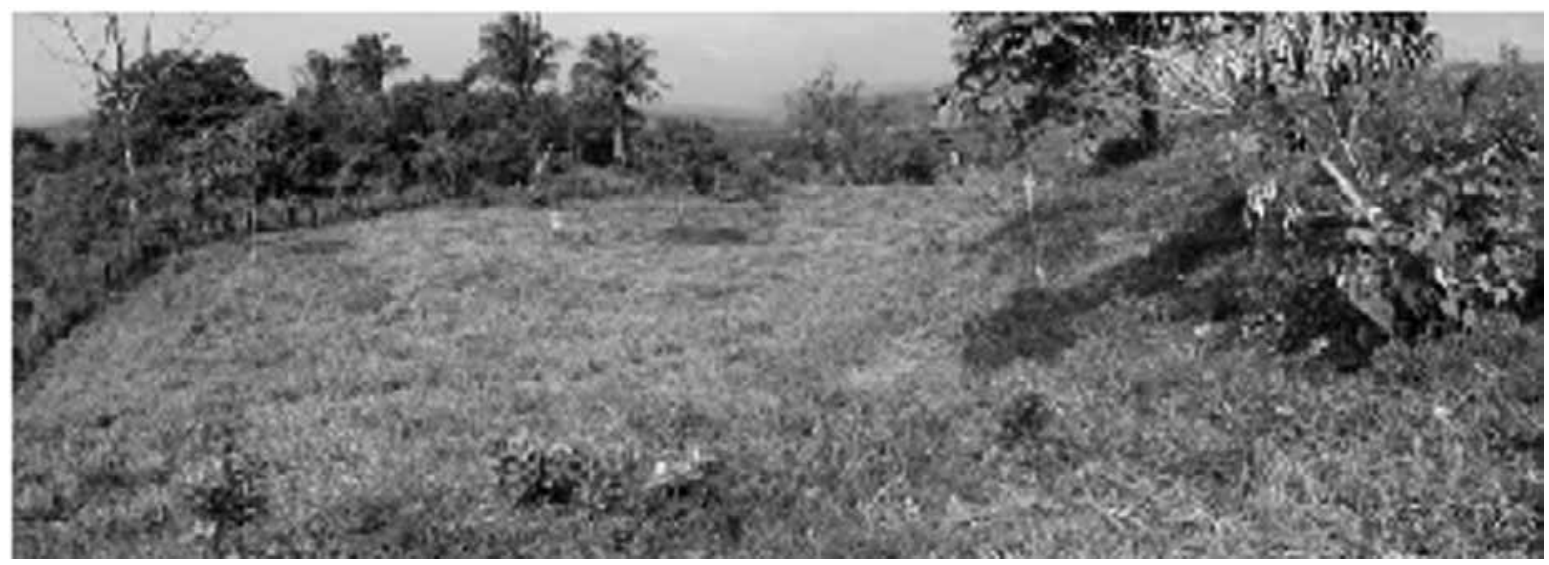




\section{Foto 3. Plaza del sitio El Chichón-Acapulco}

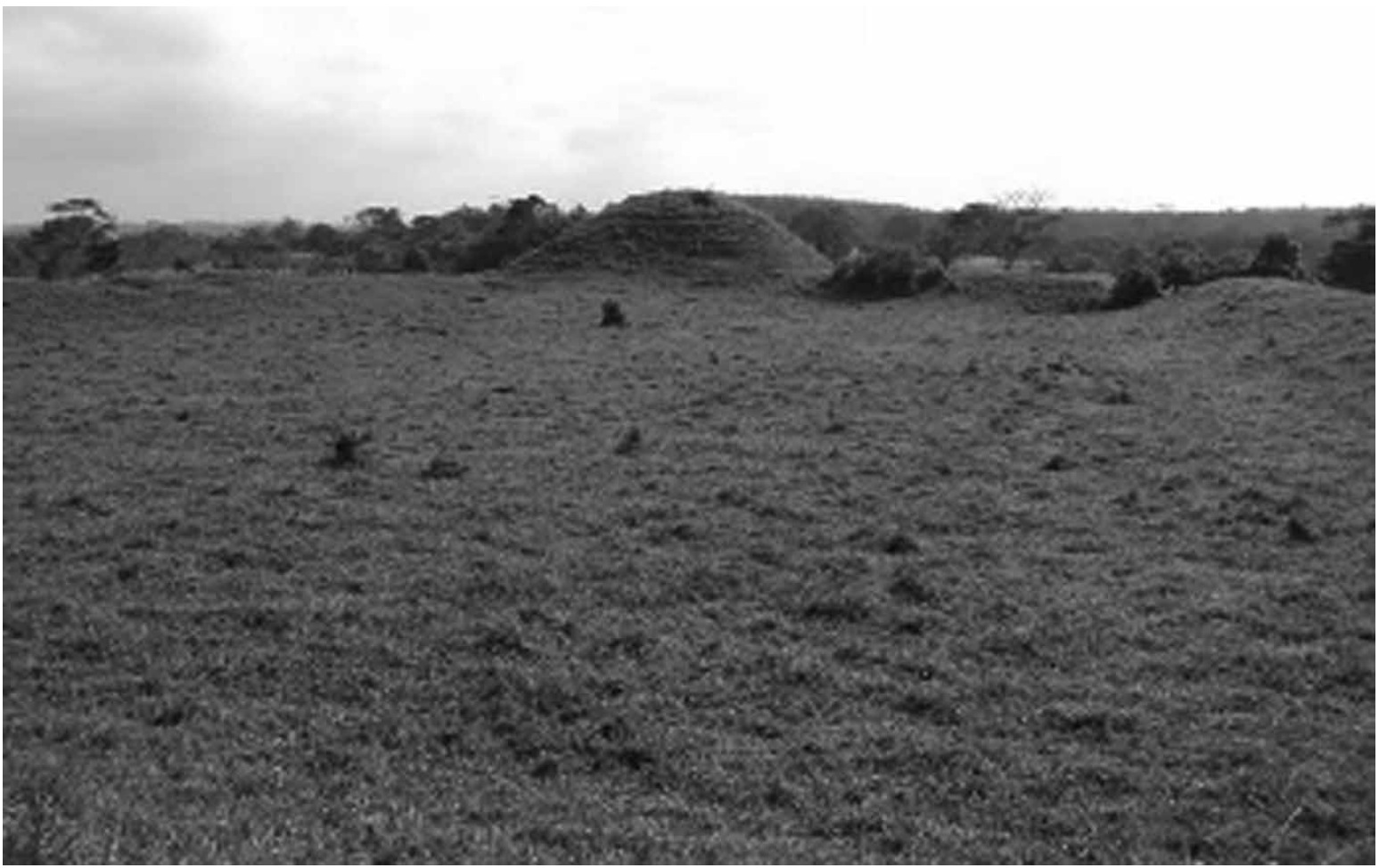

